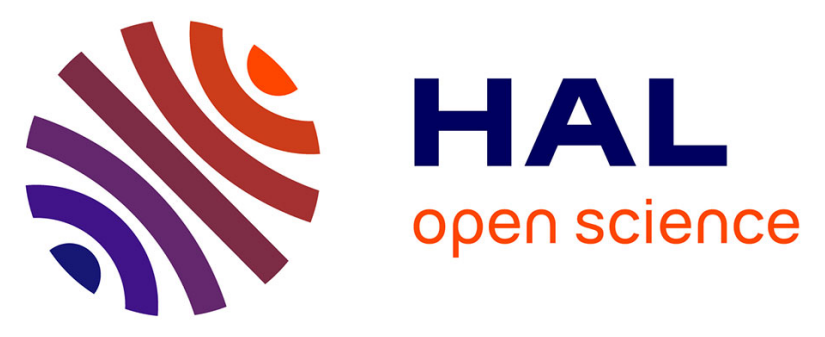

\title{
The neuronal correlates of mirror therapy: an fMRI study on mirror induced visual illusions in stroke patients
}

Marian E. Michielsen, Marion Smits, Gerard M Ribbers, Henk J Stam, Jos N van Der Geest, Johannes Bj Bussmann, Ruud W Selles

\section{To cite this version:}

Marian E. Michielsen, Marion Smits, Gerard M Ribbers, Henk J Stam, Jos N van Der Geest, et al.. The neuronal correlates of mirror therapy: an fMRI study on mirror induced visual illusions in stroke patients. Journal of Neurology, Neurosurgery and Psychiatry, 2010, 82 (4), pp.393. 10.1136/jnnp.2009.194134 . hal-00578709

\section{HAL Id: hal-00578709 \\ https://hal.science/hal-00578709}

Submitted on 22 Mar 2011

HAL is a multi-disciplinary open access archive for the deposit and dissemination of scientific research documents, whether they are published or not. The documents may come from teaching and research institutions in France or abroad, or from public or private research centers.
L'archive ouverte pluridisciplinaire HAL, est destinée au dépôt et à la diffusion de documents scientifiques de niveau recherche, publiés ou non, émanant des établissements d'enseignement et de recherche français ou étrangers, des laboratoires publics ou privés. 
1 The neuronal correlates of mirror therapy: an fMRI study on mirror induced visual illusions in 2 stroke patients

3

4 Marian E. Michielsen, MSc ${ }^{1}$

5 Marion Smits, MD, $\mathrm{PhD}^{2}$

6 Gerard M. Ribbers, MD, $\mathrm{PhD}^{1,3}$

7 Henk J. Stam, MD, PhD ${ }^{1}$

8 Jos N. van der Geest, $\mathrm{PhD}^{4}$

9 Johannes B.J. Bussmann, $\mathrm{PhD}^{1}$

10 Ruud W. Selles, PhD ${ }^{1,5}$

11

12 Department of Rehabilitation Medicine, Erasmus MC University Medical Center, Rotterdam, The 13 Netherlands

$14{ }^{2}$ Department of Radiology, Erasmus MC University Medical Center, Rotterdam, The Netherlands

$15{ }^{3}$ Rijndam Rehabilitation Center, Rotterdam, The Netherlands

$16{ }^{4}$ Department of Neuroscience, Erasmus MC University Medical Center, Rotterdam, The Netherlands

175 Department of Plastic and Reconstructive Surgery, Erasmus University Medical Center, Rotterdam,

18 The Netherlands

19

20 Address for corresponding author and reprint requests:

21

22

Marian E. Michielsen

23 Department of Rehabilitation Medicine

24 Erasmus MC University Medical Center

25 Room Ee-1622

26 PO Box 2040

273000 CE Rotterdam, The Netherlands

28

29

Telephone: +31 107044599

30 Fax: +31107033843

31 Email:m.michielsen@erasmusmc.nl

32

33

Key words: Stroke, Upper-Limbs, Rehabilitation, fMRI, Mirror Therapy

34

35 Word count: $\mathbf{3 7 0 9}$

36 
Aim

40 To investigate the neuronal basis for the effects of mirror therapy in stroke patients.

41 Methods

42 Twenty-two stroke patients participated in this study. We used functional magnetic resonance imaging

43 to investigate neuronal activation patterns in two experiments. In the unimanual experiment, patients

44 moved their unaffected hand, either while observing it directly (no-mirror condition), or while observing

45 its mirror reflection (mirror condition). In the bimanual experiment, patients moved both hands, either

46 while observing the affected hand directly (no-mirror condition) or while observing the mirror reflection

47 of the unaffected hand in place of the affected hand (mirror condition). A two-factorial analysis with

48 movement (activity versus rest) and mirror (mirror versus no mirror) as main factors was performed to

49 asses neuronal activity resultant of the mirror illusion.

\section{Results}

51 Data of 18 participants were suitable for analysis. Results showed a significant interaction effect of 52 movement $x$ mirror during the bimanual experiment. Activated regions were the precuneus and the 53 posterior cingulate cortex ( $\mathrm{p}<.05 \mathrm{FDR})$.

\section{Conclusion}

55 In this first study on the neuronal correlates of the mirror illusion in stroke patients we showed that during bimanual movement the mirror illusion increases activity in the precuneus and the posterior cingulate cortex, areas associated with awareness of the self and spatial attention. By increasing awareness of the affected limb the mirror illusion might reduce learned non-use. The fact that we did not observe mirror-related activity in areas of the motor or mirror neuron system questions popular theories that attribute the clinical effects of mirror therapy to these systems. 


\section{INTRODUCTION}

64 Mirror therapy was first introduced by Ramachandran and co-workers to alleviate phantom limb pain in amputees.[1] By showing patients the reflection of their unimpaired arm in a mirror, they retrieved the sensation of their amputated arm without pain. Since then, the paradigm of mirror therapy has also been studied in other pain related syndromes (especially chronic regional pain syndrome) [2] and motor related syndromes (stroke, hand surgery).[3,4] The focus of these studies has been on potential clinical effects; reduction of pain and improvement of motor function. Relatively little research has focussed on the mechanisms that underlie the effects of mirror therapy.

Presumably, different working mechanisms are behind the effects of mirror therapy on pain and motor symptoms. For the latter category, the focus of the current study, a number of mechanisms have been proposed. Ramachandran originally hypothesised that paralysis following stroke might have a 'learned' component, which could possibly be 'unlearned' by means of the mirror illusion.[5] Others suggested that mirror therapy might be a form of visually guided motor imagery.[6] Motor imagery itself has proven to be effective in the rehabilitation of patients with hemiparesis [7] and the mirror induced visual feedback of the imagined movement might further facilitate this. In addition, it has been hypothesized that the observation of the mirror illusion might trigger the mirror neuron system (MNS).[3] Mirror neurons were initially discovered by Di Pelligrino and co-workers in monkeys[8] and are a particular type of neurons that discharge both with performance of a motor action and with observation of another individual performing similar motor actions.[9] As single cell studies are not normally performed in the human brain, there is not yet direct evidence for the existence of a mirror neuron system in humans. However, brain imaging data do suggest the existence of a similar system.[10,11] Previous research has indicated the potential of the MNS in motor recovery by showing that the observation of movements performed by others improves motor performance in stroke patients.[12] It is conceivable that observing one's own mirrored movement promotes recovery in a similar way.

A number of studies have evaluated the neuronal correlates of mirror therapy by examining 89 the observation of the mirror reflection of a moving hand in healthy subjects. These studies were based on the hypothesis that the mirror illusion would increase excitability or activity in primary motor

91 areas in the hemisphere ipsilateral to the moving hand. Using transcranial magnetic stimulation (TMS) 92 [13-15], magnetoencephalography (MEG) [16], electroencephalography (EEG) [17] and functional 
magnetic resonance imaging (fMRI) [18] the authors compared neuronal activity or excitability 94 ipsilateral to the moving hand with or without observing its mirror reflection. The MEG study reported 95 the mirror illusion to suppress $20-\mathrm{Hz}$ activity, indicating increased activation of the primary motor 96 cortex [16], while the EEG study reported that the mirror illusion of movement induced lateralized readiness potentials, indicating cortical motor preparation for the non-moving hand.[17] On the other hand, the TMS studies either found no effect of the mirror illusion on motor cortex excitability $[13,14]$ or indicated that the mirror illusion needs to be combined with motor imagery in order to increase motor cortex excitability.[15] Finally, an fMRI study from our research group [18] found no increased activity in sensorimotor areas as a result of the mirror illusion, but did find an increase in activity in the superior temporal sulcus (STS), presumed to be due to involvement of the MNS.[19]

So far, no studies on the neuronal correlates of the mirror illusion have been performed in

104 patient groups. It seems obvious that caution has to be taken when generalizing results from mirror studies in healthy participants to stroke patients. Patients have a damaged hemisphere, and alteration of activity within that hemisphere might not be as easily achieved as in healthy participants.

107 Furthermore, stroke patients performing mirror therapy are generally instructed to practice bi-

108 manually, moving affected and un-affected limb together.[3,20,21] As stroke patients move 109 asymmetrically, placing a mirror between their hands will give them a sudden illusion of normal 110 movement of the involved hand, creating an incongruence between task performance and visual 111 feedback. This situation can not be created similarly in healthy controls, and is conceptually different 112 from the experiments in which healthy controls only move one hand.

113 In summary, while clinical trials have presented promising results of mirror therapy in several 114 patients groups, the working mechanisms have not yet been investigated in patients. Additionally, 115 results of the studies on the working mechanisms in healthy participants have not been conclusive and 116 effects that have been found in these studies cannot be generalized to stroke patients. In the present 117 study we therefore investigated the neuronal correlates of the mirror illusion in stroke patients. We 118 used fMRI to compare two different sets of conditions: 1) moving the unaffected hand while observing 119 it directly versus moving the unaffected hand while observing its mirror reflection and 2) moving both 120 hands while observing the affected hand directly versus moving both hands while observing the mirror 121 reflection of the unaffected hand in place of the affected hand. We hypothesised that observing the 122 mirror reflection would increase neuronal activity in the affected hemisphere. 


\section{Participants}

126 Patients that took part in this experiment were selected participants of a randomized controlled trial 127 (RCT) investigating the effects of a rehabilitation program of mirror therapy (www.trialregister.nl 128 NTR1052). In this trial, 40 stroke patients were included and randomly assigned to either an experimental (mirror) group or a control group. Patients from the trial that were eligible to be scanned

130 in an MRI scanner were asked to take part in the present study. This study took place ahead of the 131 start of the clinical trial, before patients had been allocated to a treatment group. Inclusion criteria for 132 the RCT were knowledge of the Dutch language, a Brunnstrom Score for the upper-extremity between

133 III and V, home dwelling status and at least one year post stroke. Patients with neglect, co-morbidities 134 that influenced upper-extremity usage or who had suffered multiple strokes were excluded from 135 participation. For patients to be able to take part in the present study, the following additional inclusion

136 criteria applied: a Brunnstrom score of IV or V and standard MRI exclusion criteria. Application of 137 these criteria resulted in a total of 22 eligible patients. The study was approved by the Medical Ethics

138 Committee of the Erasmus MC Rotterdam and all patients gave written informed consent before 139 participating in the study. Before the fMRI experiment started the Fugl Meyer [22] assessment of 140 upper-extremity function was administered to all participants for descriptive purposes.

\section{2 fMRI experiment}

143 The fMRI paradigm we used was based on one previously designed in our laboratory.[18] We 144 performed two separate experiments each involving two conditions within a single scanning session. 145 In the first experiment patients were instructed to only move their unaffected hand (unimanual), either 146 while looking directly at it (no mirror condition) or while observing its reflection in a mirror (mirror 147 condition). In the second experiment patients were instructed to move both their hands (bimanual) 148 either while looking directly at their affected hand (no mirror condition) or while observing the mirror 149 reflection of their unaffected hand in place of their affected hand (mirror condition) (see Figure 1). In all 150 four conditions, patients could see two hands. In the no mirror conditions of both experiments, patients 151 had a direct view of both their affected and their unaffected hand. In the mirror conditions, patients had 
152 a direct view of their unaffected hand and saw the reflection of their unaffected hand in place of their 153 affected hand, also leading to visual feedback of two hands.

154 During scanning, patients lay on their back in the scanner with their upper arms comfortably 155 resting on the scanner table alongside their torso and their elbows flexed in such a way that their 156 hands were 20 centimetres apart above their waists. By means of two mirrors attached to the head 157 coil above the head, patients were able to look in the direction of their feet and could thus view both 158 their hands.

159 All four conditions were performed using a block design, consisting of 10 alternating 30s 160 periods of 5 rest blocks and 5 active blocks. In the active blocks patients had to open and close either 161 their unaffected hand or both hands, in the rest blocks patients had to hold their hands still. Patients 162 were instructed to pace the opening of their hands to a metronome with a rhythm of $0.5 \mathrm{~Hz}$. The 163 onsets of the rest and active conditions were indicated verbally using simple words (start, rest) generated by a computer program (Matlab 7.1; Mathworks, Sherborn, Mass). Auditory stimuli were 165 presented to the patients through MRI compatible headphones. The hand movement was practiced before the scan session started.

167 The four conditions were presented to the patients in random order. During the mirror 168 conditions, a large mirror was placed between the subjects' hands in such a way that the mirror image 169 of the unaffected hand was superimposed on the position of the affected hand. The large mirror was 170 made of MRI-compatible material (plexiglass) and was shaped in such a way that it fitted inside the 171 scanner bore and fully obstructed the view of the hand behind the mirror (see Figure 2). In this way, a 172 visual illusion of two normal hands was created. Before the scanning session, patients practiced 173 outside the scanner with a regular mirror as used during mirror therapy in order to make sure they 174 experienced the visual illusion. While it is hard to objectively quantify the presence or strength of the 175 illusion, all subjects reported that the illusion of seeing the affected hand moving in an unimpaired 176 fashion was similar to their experience during the mirror exercises outside the MRI scanner. 177 Imaging was performed on a 3T MR system (HD platform, GE Healthcare, Milwaukee, Wis).

178 For anatomical reference, a high-resolution, 3-dimensional, inversion recovery, fast spoiled gradient 179 echo, T1-weighted image was acquired (TR/TE/TI 10.7/2.2/300 ms, $18^{\circ}$ flip angle, matrix 416×256, 180 and field of view $250 \times 175 \mathrm{~mm}^{2}$ ). For functional imaging, a single-shot, T2*-weighted, gradient echo 181 echo-planar imaging (EPI) sequence was used (TR/TE $3000 / 30 \mathrm{~ms}, 75^{\circ}$ flip angle, matrix $64 \times 96$, field 
182 of view $220 \times 220 \mathrm{~mm}^{2}$ ). An fMRI acquisition lasted for $5 \mathrm{~m} 15 \mathrm{~s}$, including 15 seconds of dummy scans

183 that were discarded. For each of the four conditions 100 volumes were collected. The imaging volume

184 covered the entire brain including the cerebellum.

185

\section{Statistical analyses}

187 The imaging data were analyzed using statistical parametric mapping software (SPM5, Wellcome

188 Department of Cognitive Neurology, University College London, UK) implemented in MATLAB version

1897.1 (Mathworks, Sherborn, MAS).

190 All functional images for each participant were realigned to the first scan of each condition and

191 then coregistered to the T1-weighted anatomical scan. Subsequently, images were transformed to

192 standard Montreal Neurologic Institute space. To prevent warping around the lesions, we used a

193 segmentation-based normalization approach.[23] Finally, normalized images were spatially smoothed

194 by using a Gaussian filter of 8-mm FWHM.

195 Preliminary analyses showed that the realignment parameters estimated during spatial 196 preprocessing were sometimes correlated with the task design. Therefore, we decided not to model

197 the realignment parameters in the design matrix as regressors of no interest, as this would have 198 resulted in cancelling out task-related activation. Instead, we used the ArtRepair Toolbox [24], which 199 evaluates all volumes, detects the ones most affected by movement, and deweights these in the 200 general linear model estimation. The experimental block design was convolved with the canonical 201 hemodynamic response function, and the resulting model was estimated using a high-pass filter at $202128 \mathrm{~s}$ in order to remove low-frequency artifacts.

203 In the first-level analysis, statistical maps were calculated for each of the four task blocks (i.e.

204 movement with mirror, movement without mirror, rest with mirror, rest without mirror) for each patient 205 and each experiment (bimanual and unimanual) separately. Statistical maps of patients with left-sided 206 lesions were flipped about the midsagittal plane, so that the affected hemisphere corresponded to the 207 right side of the brain for all patients. The statistical maps were used for second level analyses. 
211 We performed a two factorial analysis with movement (activity versus rest) and mirror (mirror versus

212 no mirror) as main factors for both the unimanual and the bimanual experiments separately. Main

213 effects of movement and mirror as well as the interaction between movement and mirror were

214 investigated. Significance was set at $p<.05$ (FDR corrected) with a minimum cluster size of 20.

216 RESULTS

217 Four patients were discarded from further analysis, one due to scanner failure, and three due to 218 scanner artefacts in their data sets. The remaining analyses were thus conducted using data sets of 21918 patients. The characteristics of these patients are presented in Table 1.

220

Table 1

222 Patient characteristics of the study participants

\begin{tabular}{|c|c|c|}
\hline Characteristic & Total $^{*}$ & 223 \\
\hline No. of patients & 18 & 225 \\
\hline Age (years) & $54.7 \pm 9.9$ & 226 \\
\hline Time since stroke (years) & $5.2 \pm 3.6$ & 227 \\
\hline Sex (male/female) & $10 / 8$ & 228 \\
\hline Affected side dominant/nondominant) & $6 / 12$ & 229 \\
\hline Type of stroke (infarct/haemorrhage) & $16 / 2$ & 230 \\
\hline Location of stroke lesion & & 231 \\
\hline - Cortical (with or without subcortical) & 13 & 232 \\
\hline - Subcortical & 3 & 233 \\
\hline - Brainstem ${ }^{\dagger}$ & 2 & 234 \\
\hline FM score & $41.9 \pm 11$ & 3235 \\
\hline
\end{tabular}

237 *Values are mean \pm standard deviation

$238{ }^{\dagger}$ Brainstem lesions are located in the pons, above the crossing of the cortico-spinal tract

In a two-factorial design, we examined main effects of movement (task condition versus rest),

241 mirror (mirror condition versus no mirror condition), and the interaction effect between movement and 242 mirror for both experiments. Observed activation patterns for the main effect of movement were in 243 accordance with the expected activation for uni- and bimanual hand motor tasks. Activity was 244 observed in the pre- and postcentral gyrus (primary motor and sensory cortex), the medial superior 
245 frontal gyrus (SMA), at the junction of the superior frontal sulcus and the precentral sulcus (premotor

246 cortex) and in the cerebellum.

247

248 Table 2

249 Areas of activation (unimanual experiment: main effect of movement)

\begin{tabular}{|c|c|c|c|c|c|c|}
\hline \multirow[t]{2}{*}{ Anatomical location } & \multirow[t]{2}{*}{ Side } & \multirow[t]{2}{*}{ Cluster size } & \multirow[t]{2}{*}{ Z-score } & \multicolumn{3}{|c|}{ MNI } \\
\hline & & & & $\mathbf{x}$ & $\mathbf{y}$ & $\mathbf{z}$ \\
\hline Pre- and Post central Gyrus & Unaffected & 2103 & 7.57 & -34 & -26 & 54 \\
\hline Cerebellum & Unaffected & 1287 & 7.28 & 16 & -56 & -20 \\
\hline Middle Occipital/Temporal gyrus & Affected & 612 & 6.18 & 42 & -64 & 4 \\
\hline Medial Frontal Gyrus, Superior & Affected, & & & & & \\
\hline Frontal Gyrus, Cingulate Gyrus & Unaffected & 448 & 6.17 & -6 & -6 & 56 \\
\hline Middle Occipital/Temporal gyrus & Unaffected & 491 & 5.98 & -48 & -70 & -2 \\
\hline Precentral Gyrus & Unaffected & 36 & 5.72 & -52 & 0 & 8 \\
\hline
\end{tabular}

250

251 
252 Areas of activation (bimanual experiment: main effect of movement)

\begin{tabular}{|c|c|c|c|c|c|c|}
\hline \multirow[t]{2}{*}{ Anatomical location } & \multirow[t]{2}{*}{ Side } & \multirow[t]{2}{*}{ Cluster size } & \multirow[t]{2}{*}{ Z-score } & \multicolumn{3}{|c|}{ MNI } \\
\hline & & & & $\mathbf{x}$ & y & $\mathbf{z}$ \\
\hline Pre- and Post central Gyrus & Unaffected & 853 & 7.11 & -42 & -20 & 52 \\
\hline $\begin{array}{l}\text { Medial Frontal Gyrus, Superior } \\
\text { Frontal Gyrus, Cingulate Gyrus }\end{array}$ & Unaffected & 331 & 6.43 & -6 & -4 & 58 \\
\hline Pre- and Post Central Gyrus, & & & & & & \\
\hline Medial and Superior Frontal & Affected & 665 & 6.36 & 36 & -16 & 56 \\
\hline Gyrus, Cingulate Gyrus & & & & & & \\
\hline $\begin{array}{l}\text { Middle Occipital gyrus, } \\
\text { Inferior Temporal gyrys }\end{array}$ & Affected & 33 & 5.63 & 44 & -72 & 0 \\
\hline Middle Occipital/Temporal & & & & & & \\
\hline gyrus, Inferior Temporal gyrys & Unaffected & 55 & 5.35 & -46 & -68 & 0 \\
\hline
\end{tabular}

253

254 Areas are tresholded at $\mathrm{p}<.05$ (FDR corrected) with a minimum cluster size of 20 voxels. $\mathrm{MNI}=$

255 Montreal Neurological Institute.

256

257 Analysis of the main effect of mirror (mirror versus no mirror) showed no significant areas of 258 activation in either of the two experiments. The interaction of mirror $x$ movement showed no significant 259 activation for the unimanual experiment but it did show significant activation in the bimanual 260 experiment; in the precuneus and the posterior cingulate cortex. Post-hoc analysis revealed that this 261 was caused by increased activity in the movement with mirror condition versus the movement without 262 mirror condition. Table 3 and Figure 3 show the activated clusters for the interaction effect of 263 movement $\mathrm{x}$ mirror in the bimanual experiment. 
Table 3

266 Areas of activation (bimanual experiment: interaction movement $\mathbf{x}$ mirror)

\begin{tabular}{llccccc}
\hline Anatomical location & Side & Cluster size & Z-score & & MNI & \\
& & & $\mathbf{x}$ & $\mathbf{y}$ & $\mathbf{z}$ \\
\hline & Affected, & & & & & \\
Precuneus & Unaffected, & 78 & 5.28 & 2 & -58 & 58 \\
& Central & & & & & \\
& & & & & & \\
& & & & & & \\
Posterior Cingulate Cortex & Unaffected & 117 & 4.68 & -6 & -36 & 8 \\
\hline
\end{tabular}

267

268 Areas are tresholded at $\mathrm{p}<.05$ (FDR corrected) with a minimum cluster size of 20 voxels. $\mathrm{MNI}=$

269 Montreal Neurological Institute.

270

271 DISCUSSION

272 In an attempt to unravel the working mechanism of mirror therapy, this study investigated the neuronal

273 correlates of the mirror-induced visual illusion in stroke patients. In this first study, to our knowledge, in

274 which stroke patients participated instead of healthy volunteers, we showed increased activity as a

275 result of the mirror illusion during bimanual movement in two areas: the precuneus and the posterior

276 cingulate cortex. We found no differential effect on neuronal activity of the mirror illusion during

277 unimanual movement, nor did we find evidence for the mirror illusion to increase activity in motor

278 areas or the mirror neuron system.

279 A network including both the precuneus and posterior cingulate cortex is reported to be 280 associated with mental representation of the self.[25] More specifically, research showed that the

281 precuneus is activated when actions are being interpreted as being controlled by the self as well as

282 during self centred mental imagery strategies [26] whereas the cingulate cortex becomes activated

283 during spatial navigation [27] and has been found to process information about the spatial positions of

284 the limbs in monkeys.[28] The mirror illusion of a normal moving affected hand thus seems to increase

285 alertness and spatial attention towards this hand. The fact that we did not find this activation during the

286 unimanual condition, suggests that it is not so much the illusion of a virtual moving hand that causes

287 this activation, but the mismatch between the movement one performs and the movement that is 
observed. Research showing that the cingulate cortex becomes activated during conflict monitoring, specifically during action conflicts, supports this.[29]

Two previous studies in healthy participants have reported increased neuronal activity in similar areas as a result of an incongruence between movement observation and action.[30-32] Dohle et al.[32] found that such incongruence increased activation in occipital and posterior parietal areas, amongst them the precuneus, which they suggest to play a decisive role during mirror therapy.[21] In an earlier experiment Fink et al.[30] also reported on the neural correlates of a conflict between visual and proprioceptive information. Using positron emission tomography (PET) they investigated the effect of the mirror illusion during either in-phase movements or out-of-phase movements that are perceived as in-phase movements due to the mirror. Their results showed an effect of the mirror in the dorsolateral prefrontal cortex (DLPCF) and in the superior posterior parietal cortex (Brodmann's area (BA) 7). BA 7 is a point of convergence between vision and proprioception and plays a role in visuomotor coordination. The precuneus, which in our study became activated during the bimanual mirror condition, is part of BA 7 [26] but is located more medial than the area of activation reported by Fink et al. The main difference between our study and Fink et al.'s is that in the latter participants actively had to create the motor-sensory conflict (by moving out-of-phase), while in our patient group this resulted from the involved arm not being able to perform similar movements as the non-involved arm. The situation created by Fink et al. likely induces a larger cognitive burden for the participants, which may explain increased DLPCF activation, while the main effect of the mirror they observed in BA 7 is in line

307 with our findings of precuneus activation in the presence of a motor-sensory conflict, albeit under different experimental settings.

The question is how the involvement of the areas we found activated by the mirror illusion relates to the reported improvements in motor function following mirror therapy.[3,21] Possibly, by

311 increasing the spatial attention towards the affected limb the mirror illusion might help in overcoming 312 the learned non-use phenomenon [21,33], and as a result of the ensuing increased use of the limb 313 improve motor performance. An alternative hypothesis, supported by the fact that we only observed 314 increased cortical activation during the bimanual experiment, might be that the effects of the mirror lie 315 in an enhancement of spatial coupling between limbs. It is well known that when two arms move 316 simultaneously, movements become more temporally and spatially stable.[34] In stroke rehabilitation, 317 this phenomenon has been exploited in the form of bimanual training programs. Several studies have 
318 shown that bimanual training strategies have a favourable effect over unimanual training [35], 319 demonstrating that spatial coupling can cause the affected limb to take on the properties of the non320 effected limb, thereby improving motor performance. The hypothesis that the mirror illusion enhances 321 this spatial coupling is supported by studies on healthy volunteers, showing that the mirror illusion 322 increased the tendency of one limb to take on the spatial properties of the other limb.[36,37]

323 So far, several reviews and clinical studies have attributed the effects of mirror therapy to 324 activation of motor areas or the mirror neuron system (MNS).[3,38-40] As mentioned, in the present 325 study we did not observe activation resulting from the mirror illusion in these areas (e.g. M1, PMC, 326 SMA, Broca's area), neither in the unimanual experiment nor in the bimanual experiment. Research 327 performed in healthy subjects has so far also been unable to provide convincing evidence for the 328 activation of these areas by the mirror illusion, only the fMRI study of Matthys et al. showed some 329 evidence for MNS activation by reporting increased activation within STS.[18] However, although STS 330 is reported to be related to the MNS [19], this area has been associated with many different 331 behaviours and its exact function remains poorly understood.[41] Matthys et al. also reported 332 activation in the superior occipital gyrus, an area connected with the PPC trough the dorsal stream. 333 Activation of this area may reflect increased attentional demands for the integration of vision and 334 proprioception induced by the mirror, which is in line with our present results. It has to be noted that 335 the analysis strategy of Matthys et al. differed from the strategy employed in the present paper. 336 Contrary to our approach, Matthys et al. did not apply an ANOVA design and thus did not examine the 337 effect of the mirror, the hand motor performance and its interaction separately. As a final note on the 338 proposed activation of the MNS or motor system by the mirror illusion, a previous study showed that 339 whereas observation of actions attributed to another individual activated the motor system, 340 observation of identical actions linked to the self did not.[42] The MNS thus seems to distinguish 341 between observing actions linked to the self and actions linked to others. This finding further 342 undermines the notion that the mirror illusion of self-performed movements might trigger the motor or 343 mirror neuron system.

344 We acknowledge that our study has some limitations. In general, detecting study-related 345 effects in fMRI experiments in stroke patients is difficult as the neuronal circuitry may be distorted and 346 heterogeneous between subjects. Some authors try to get around this issue by including only patients 347 with minor lesions, which is a major source of selection bias. In the current study, in order to enlarge 
348 the contrast between mirror and no mirror conditions in the bimanual experiment, we explicitly enrolled 349 patients with larger motor deficits. Consequently, the within-group variability was considerable, which 350 may have decreased the power to detect differences between conditions. Another issue, related to the 351 severity of their motor deficit, is that some patients had problems keeping their head still during the 352 experimental task. As these movements were task-related, we could not simply regress out head 353 motion-related activation as this would have cancelled out task-related activation as well. However, we 354 used an alternative, sensitive method to deal with this issue (see Methods section), and activation 355 patterns we observed are in accordance with the expected activation for uni- and bimanual hand 356 motor tasks, implicating the validity of our analysis. A general limitation of this and similar studies is 357 that it is difficult to objectify the strength of the illusion patients experience when inside the scanner. To 358 maximize the mirror illusion during scanning, all patients practiced with a standard mirror used for 359 mirror therapy outside the scanner, and they all reported similar illusion strength during the 360 measurements inside the scanner as outside.

361 In conclusion, the present study showed that during bimanual movement, the mirror illusion 362 alters neuronal activation in the precuneus and posterior cingulate cortex, areas related to alertness 363 and spatial-awareness. We found no differential effect on neuronal activity of the mirror illusion during 364 unimanual movements, nor did we find evidence for the mirror illusion to increase activity in motor 365 areas. By increasing awareness of the affected limb, possibly due the mismatch between action and 366 observation, the mirror illusion might reduce learned non-use. The fact that we did not observe any 367 activation in areas belonging to the motor or mirror neuron system questions popular theories that 368 contribute the clinical effects of mirror therapy to these systems. As research into the working 369 mechanism of mirror therapy has so far mainly focussed on these systems, we suggest that future 370 research should adopt a broader perspective, amongst other things taking the ideas as proposed in 371 this paper into account. Since a better understanding of why and how mirror therapy works may lead 372 to a more effective application and might help in selecting patients for which mirror therapy will be 373 most effective, it is important that efforts to unravel the neuronal correlates of mirror therapy continue. 374 


\section{ACKNOWLEDGMENTS}

376 We want to thank Martine Eckhardt for her help with the measurements and organization of the study

377 and Maarten Bobbert, Daphne Heemskerk and David Warmerdam for their help with the analysis of

378 the data and interpretation of results. Also, we would like to acknowledge the two anonymous

379 reviewers for their valuable suggestions on the data analysis and the manuscript. This study was

380 supported by Hersenstichting Nederland, Fonds NutsOhra, Innovatiefonds Zorgverzekeraars, Prinses

381 Beatrix Fonds and Wetenschappelijk College Fysiotherapie.

382

383 Competing Interest: None declared

384 


\section{COPYRIGHT LICENCE STATEMENT}

386 The Corresponding Author has the right to grant on behalf of all authors and does grant on behalf of

387 all authors, an exclusive license (or non-exclusive for government employees) on a worldwide basis to

388 the BMJ Publishing Group Ltd, and its Licensees to permit this article (if accepted) to be published in

389 Journal of Neurology, Neurosurgery \& Psychiatry and any other BMJPGL products and to exploit all

390 subsidiary rights, as set out in our license.

391 
393 [1] Ramachandran VS, Rogers-Ramachandran D, Cobb S. Touching the phantom limb. Nature. 3941995 Oct 12;377(6549):489-90.

395 [2] McCabe CS, Haigh RC, Ring EF, Halligan PW, Wall PD, Blake DR. A controlled pilot study of 396 the utility of mirror visual feedback in the treatment of complex regional pain syndrome (type 1). 397 Rheumatology (Oxford). 2003 Jan;42(1):97-101.

398 [3] Yavuzer G, Selles R, Sezer N, Sutbeyaz S, Bussmann JB, Koseoglu F, et al. Mirror therapy 399 improves hand function in subacute stroke: a randomized controlled trial. Arch Phys Med Rehabil. 4002008 Mar;89(3):393-8.

401 [4] Rosen B, Lundborg G. Training with a mirror in rehabilitation of the hand. Scandinavian journal 402 of plastic and reconstructive surgery and hand surgery / Nordisk plastikkirurgisk forening [and] Nordisk 403 klubb for handkirurgi. 2005;39(2):104-8.

404 [5] Ramachandran VS. Phantom limbs, neglect syndromes, repressed memories, and Freudian 405 psychology. International review of neurobiology. 1994;37:291-333; discussion 69-72.

406 [6] Stevens JA, Stoykov ME. Using motor imagery in the rehabilitation of hemiparesis. Arch Phys 407 Med Rehabil. 2003 Jul;84(7):1090-2.

408 [7] Sharma N, Pomeroy VM, Baron JC. Motor imagery: a backdoor to the motor system after 409 stroke? Stroke. 2006 Jul;37(7):1941-52.

410 [8] di Pellegrino G, Fadiga L, Fogassi L, Gallese V, Rizzolatti G. Understanding motor events: a 411 neurophysiological study. Exp Brain Res. 1992;91(1):176-80.

412 [9] Rizzolatti G, Craighero L. The mirror-neuron system. Annual review of neuroscience. 413 2004;27:169-92.

414 [10] lacoboni M, Woods RP, Brass M, Bekkering H, Mazziotta JC, Rizzolatti G. Cortical 415 mechanisms of human imitation. Science. 1999 Dec 24;286(5449):2526-8.

416 [11] Rizzolatti G, Fogassi L, Gallese V. Neurophysiological mechanisms underlying the 417 understanding and imitation of action. Nature reviews. 2001 Sep;2(9):661-70.

418 [12] Ertelt D, Small S, Solodkin A, Dettmers C, McNamara A, Binkofski F, et al. Action observation 419 has a positive impact on rehabilitation of motor deficits after stroke. Neuroimage. 2007;36 Suppl $420 \quad$ 2:T164-73.

421 [13] Garry MI, Loftus A, Summers JJ. Mirror, mirror on the wall: viewing a mirror reflection of 422 unilateral hand movements facilitates ipsilateral M1 excitability. Exp Brain Res. 2005 May;163(1):11842322.

424 [14] Funase K, Tabira T, Higashi T, Liang N, Kasai T. Increased corticospinal excitability during 425 direct observation of self-movement and indirect observation with a mirror box. Neuroscience letters. 4262007 May 29;419(2):108-12.

427 [15] Fukumura K, Sugawara K, Tanabe S, Ushiba J, Tomita Y. Influence of mirror therapy on 428 human motor cortex. The International journal of neuroscience. $2007 \mathrm{Jul} ; 117(7): 1039-48$.

429 [16] Tominaga W, Matsubayashi J, Deguchi Y, Minami C, Kinai T, Nakamura M, et al. A mirror 430 reflection of a hand modulates stimulus-induced $20-\mathrm{Hz}$ activity. Neuroimage. 2009 Jun;46(2):500-4. 
431 [17] Touzalin-Chretien P, Dufour A. Motor cortex activation induced by a mirror: evidence from 432 lateralized readiness potentials. J Neurophysiol. $2008 \mathrm{Jul} ; 100(1): 19-23$.

433 [18] Matthys K, Smits M, Van der Geest JN, Van der Lugt A, Seurinck R, Stam HJ, et al. Mirror434 induced visual illusion of hand movements: a functional magnetic resonance imaging study. Arch Phys 435 Med Rehabil. 2009 Apr;90(4):675-81.

436 [19] lacoboni M, Dapretto M. The mirror neuron system and the consequences of its dysfunction. 437 Nature reviews. 2006 Dec;7(12):942-51.

438 [20] Altschuler EL, Wisdom SB, Stone L, Foster C, Galasko D, Llewellyn DM, et al. Rehabilitation 439 of hemiparesis after stroke with a mirror. Lancet. 1999 Jun 12;353(9169):2035-6.

440 [21] Dohle C, Pullen J, Nakaten A, Kust J, Rietz C, Karbe H. Mirror Therapy Promotes Recovery 441 From Severe Hemiparesis: A Randomized Controlled Trial. Neurorehabil Neural Repair. 2008 Dec 12.

442 [22] Fugl-Meyer AR, Jaasko L, Leyman I, Olsson S, Steglind S. The post-stroke hemiplegic patient. 443 1. a method for evaluation of physical performance. Scandinavian journal of rehabilitation medicine. 444 1975;7(1):13-31.

445 [23] Crinion J, Ashburner J, Leff A, Brett M, Price C, Friston K. Spatial normalization of lesioned 446 brains: performance evaluation and impact on fMRI analyses. Neuroimage. 2007 Sep 1;37(3):866-75.

447 [24] Mazaika P, Whitfield-Gabrieli, S., Reiss, A. Artifact Repair for fMRI Data from High Motion 448 Clinical Subjects. Human Brain Mapping 2007; Chigago; 2007.

449 [25] Fransson P, Marrelec G. The precuneus/posterior cingulate cortex plays a pivotal role in the 450 default mode network: Evidence from a partial correlation network analysis. Neuroimage. 2008 Sep $451 \quad 1 ; 42(3): 1178-84$.

452 [26] Cavanna AE, Trimble MR. The precuneus: a review of its functional anatomy and behavioural 453 correlates. Brain. 2006 Mar;129(Pt 3):564-83.

454 [27] Moffat SD. Aging and spatial navigation: what do we know and where do we go? 455 Neuropsychology review. 2009 Dec;19(4):478-89.

456 [28] Bakola S, Gamberini M, Passarelli L, Fattori P, Galletti C. Cortical Connections of Parietal 457 Field PEc in the Macaque: Linking Vision and Somatic Sensation for the Control of Limb Action. Cereb 458 Cortex. Feb 22.

459 [29] Botvinick MM, Braver TS, Barch DM, Carter CS, Cohen JD. Conflict monitoring and cognitive 460 control. Psychological review. $2001 \mathrm{Jul} ; 108(3): 624-52$.

461 [30] Fink GR, Marshall JC, Halligan PW, Frith CD, Driver J, Frackowiak RS, et al. The neural 462 consequences of conflict between intention and the senses. Brain. 1999 Mar;122 ( Pt 3):497-512.

463 [31] Leube DT, Knoblich G, Erb M, Kircher TT. Observing one's hand become anarchic: an fMRI 464 study of action identification. Consciousness and cognition. 2003 Dec;12(4):597-608.

465 [32] Dohle C, Kleiser R, Seitz RJ, Freund HJ. Body scheme gates visual processing. J 466 Neurophysiol. 2004 May;91(5):2376-9.

467 [33] Taub E. Somatosensory deafferentation research with monkeys: implications for rehabilitation 468 medicine. In: Wilkins W, ed. Behavioral Psychology in Rehabilitation Medicine: Clinical Applications. 469 Baltimore 1980:371-401. 
470 [34] Kelso JA, Holt KG, Rubin P, Kugler PN. Patterns of human interlimb coordination emerge from 471 the properties of non-linear, limit cycle oscillatory processes: theory and data. Journal of motor 472 behavior. $1981 \mathrm{Dec} ; 13(4): 226-61$.

473 [35] Stoykov ME, Corcos DM. A review of bilateral training for upper extremity hemiparesis. 474 Occupational therapy international. 2009;16(3-4):190-203.

475 [36] Altschuler EL. Interaction of vision and movement via a mirror. Perception. 2005;34(9):1153-5.

476 [37] Franz EA, Packman T. Fooling the brain into thinking it sees both hands moving enhances 477 bimanual spatial coupling. Exp Brain Res. 2004 Jul;157(2):174-80.

478 [38] Ramachandran VS, Altschuler EL. The use of visual feedback, in particular mirror visual 479 feedback, in restoring brain function. Brain. 2009 Jul;132(Pt 7):1693-710.

480 [39] Ezendam D, Bongers RM, Jannink MJ. Systematic review of the effectiveness of mirror 481 therapy in upper extremity function. Disability and rehabilitation. 2009 May 20:1-15.

482 [40] Rizzolatti G, Fabbri-Destro M, Cattaneo L. Mirror neurons and their clinical relevance. Nature 483 clinical practice. 2009 Jan;5(1):24-34.

484 [41] Hein G, Knight RT. Superior temporal sulcus--It's my area: or is it? J Cogn Neurosci. 2008 485 Dec;20(12):2125-36.

486 [42] Schutz-Bosbach S, Mancini B, Aglioti SM, Haggard P. Self and other in the human motor 487 system. Curr Biol. 2006 Sep 19;16(18):1830-4.

488

489 
Figure 1

491 Schematic representation of the four conditions. FE indicates which hands perform the flexion-

492 extension movements, and the arrow is used to indicate the direction of gaze in each condition. 493 


\section{$494 \quad$ Figure 2}

495 Participant lying in the scanner during the mirror condition. The unaffected hand is not visible, as it is 496 positioned in front of the mirror. 


\section{$497 \quad$ Figure 3}

498 Activation map of the interaction effect of movement $x$ mirror for the bimanual experiment $(p<.05$, FDR 499 corrected, minimum cluster size 20). Label A: posterior cingulate cortex; Label B: precuneus. 
Unimanual experiment

Mirror condition

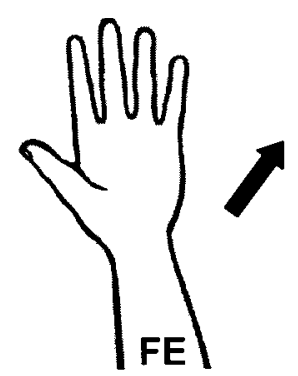

Unaffected side
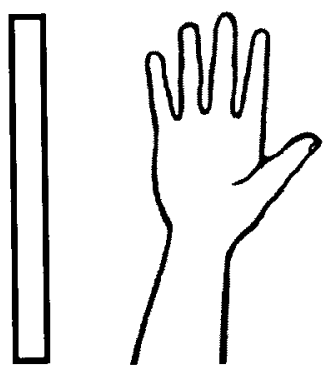

Affected side
No mirror condition

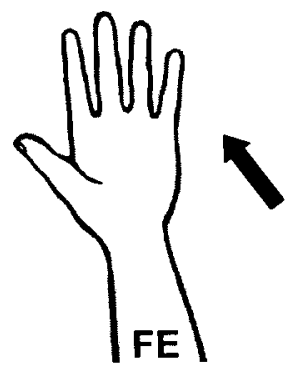

Unaffected side

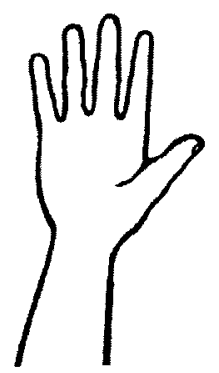

Affected side

\section{Bimanual experiment}

Mirror condition

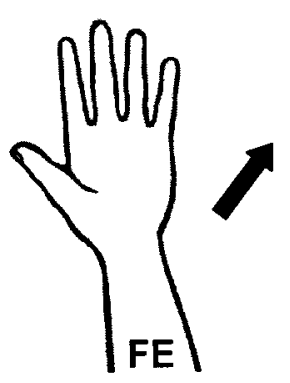

Unaffected side
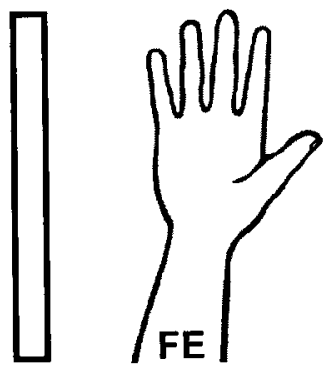

Affected side

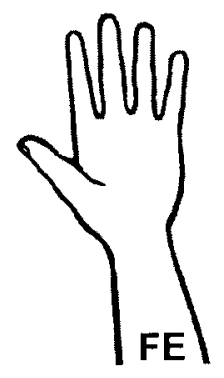

Unaffected side

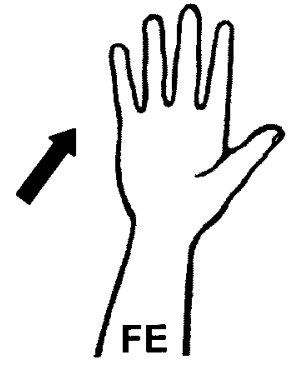

Affected side 


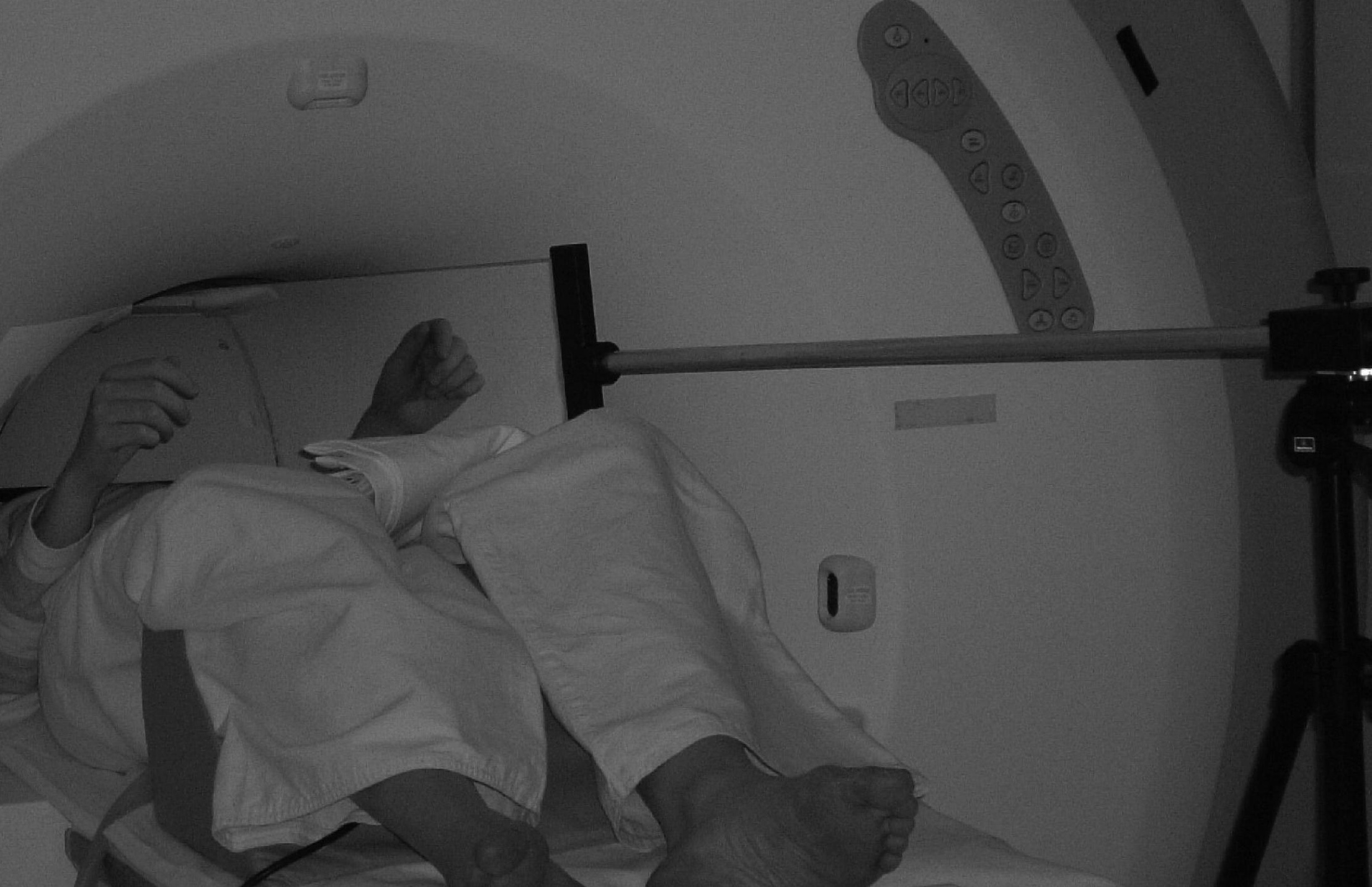




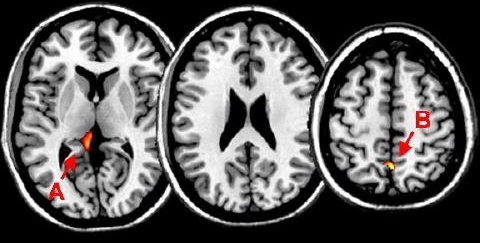

